\title{
Reviewer 2019
}

Financial Reporting would like to thank the following reviewers for their help during 2019: Michele Fabrizi (Università Padova); Roffia Paolo (Università Verona); Matthew Hoag (Gonzaga University); Velia Cenciarelli (Università Cattolica Milano); Lucrezia Songini (Università Piemonte Orientale); Paola Ramassa (Università Genova); Pietro Bonetti (IESE); Elisa Rita Ferrari (Università Kore Enna); Gaia Melloni (Lausanne University); Matteo Palmaccio (Università di Cassino e del Lazio Meridionale); Fabrizio Granà (LUISS); Rosa Lombardi (Università Sapienza); Giovanna Michelon (Bristol University); Paola Paglietti (Università Catania); Andrea Menini (Università Padova); Francesca Rossignoli (Università Verona); Giulia Leoni (Università Genova); Giorgio Mion (Università Verona); Albero Quagli (Università Genova); Andrea Lionzo (Università Cattolica Milano); Giuseppe Marzo (Università Ferrara); Sandro Sandri (Università Bologna); Mara Cameran (Università Bocconi); Domenico Campa (International University of Monaco); Claudio Teodori (Università Brescia); Patrizia Riva (Università Piemonte Orientale); Alessandra Allini (Università Federico II Napoli); Marco Allegrini (Università Pisa); Carlo Marcon (Ca’ Foscari Università Venezia); Christian Favino (Università Foggia); Simone Terzani (Università Perugia); Amedeo Pugliese (Università Padova); Riccardo Cimini (Università della Tuscia); Lorenzo Dal Maso (ESSEC); Riccardo Stacchezzini (Università Verona); Paola Demartini (Università Roma Tre); Marco Bisogno (Università Salerno); Alessandro Giannozzi (Università Firenze); Marco Pisani (Università Aquila); Alberto Incollingo (Università della Campania); Francesca Manes Rossi (Università Salerno); Olimpia Meglio (Università Sannio); Marco Fazzini (Università Europea Roma); Marco Bellucci (Università Firenze); Paola Ramassa (Università Genova); Paola Rossi (Università Trieste); Marco Giuliani (Università Politecnica delle Marche); Marco Maria Mattei (Università Bologna); Andrea Menini (Università Padova); Francesco Mazzi (Università Firenze).

We are in your debt.

Best Reviewer 2019: Riccardo Cimini (Università della Tuscia).

DOI: $10.3280 /$ FR2020-001007 